\title{
Neuron Specific Enolase (NSE): A Valuable Prognostic Factor of Central Nervous System Dysfunction Following Cardiac Surgery
}

\author{
E.A. Konstantinou, K. Venetsanou, A.P. Mitsos, K.V. Mamoura, \\ E.E. Theodosopoulou, T. Fotis, G. Baltopoulos \\ Evgenidion University Hospital, National and Kapodistrian University of Athens, \\ Faculty of Nursing, Athens, Greece
}

\begin{abstract}
Purpose: The aim of this study was to evaluate neuron specific enolase (NSE) as prognostic factor for CNS disorders developed in relation to cardiac surgery.

Patients and methods: A cohort of 92 patients were divided into two groups; the experimental group consisted of patients undergoing openheart bypass surgery (50 patients) and the control group consisting of 42 patients undergoing general surgery procedures. The blood levels of NSE were measured in both groups before and $24 \mathrm{~h}$ after the operation and have been related to the incidence of the postoperative brain damage as well as to the duration of the extracorporeal circulation (ECC).

Results: The analysis of our results showed that the changes of blood NSE levels, before and immediately after ECC in open-heart surgery have been related to the incidence of postoperative brain dysfunction according to a specific mathematical equation. This incidence was raised also in relation to the age of the patient and the duration of ECC. On the contrary, NSE seems to have no prognostic value in general surgery.
\end{abstract}

Correspondence to: Evangelos A. Konstantinou, 18 Kivelis Street, 15238, Halandri, Athens, Greece. E-mail: ekonstan30@yahoo.com
Conclusion: NSE can be used in cardiac surgery as a simple and reliable prognostic factor to predict postoperative brain dysfunction.

Key words: Neuron specific enolase (NSE); prognosis; postoperative CNS disorders; cardiac surgery

\section{INTRODUCTION}

Enolase (2-phospho-D-glycerate hydrolase) is a glycolytic enzyme expressed by several isoenzymatic dimmers $(\alpha \alpha$, $\alpha \beta, \alpha \gamma, \beta \beta, \gamma \gamma)$ composed of three distinct subunits $(\alpha, \beta$, $\gamma)$. It was first described by Moore and McGregor in 1965 . The $\alpha$-subunit is widely distributed in mammalian tissues and glial cells of the brain, while the $\beta$-subunit is localised primarily in the skeletal muscle and the myocardial tissue. Three different isoenzymes have been found in human brain: $\alpha \alpha, \alpha \gamma$ and $\gamma \gamma$, with a molecular weight of approximately 80,000 daltons each. The $\alpha \gamma$ and $\gamma \gamma$ isoforms are known as neuron specific enolase (NSE) because they initially were found in neurons and neuroendocrine cells. The $\alpha \alpha$-isoenzyme is also a dimmer and was designated as non-neuronal enolase. There are distinguished differences in biochemical properties between these two isoforms from brain tissue; most important of these differences is apparently the complete lack of immunological cross reactivity, which has been used for the quantification of NSE by several immunoassays. NSE's isoelectric point is 4.7 and is characterised by chloride, urea and temperature stability. It is assumed that NSE, being an enzyme from the cellular cytoplasm, is released during cell destruction. Thus, its presence in the 
circulation is related to a high death rate of cells with neuroendocrine differentiation, a characteristic of tumours producing the enzyme [Zomzely-Neurath, 1983]. This specific identification of NSE in blood has been extensively used to detect small cell lung cancer, neuroblastoma [Massaron et al, 1998; Zeltzer et al, 1986], neuroendocrine tumours [Ishguro et al, 1983], Creutzfeldt-Jacob disease [Zerri et al, 1995], malignant melanoma [Lorenz et al, 1989], seminoma [Fossa et al, 1992], Merkel cell carcinoma [Chang et al, 1998], medulloblastoma [Zeltzer et al, 1986], retinoblastoma [Ke Ping et al, 1995] and Guillain Barre syndrome [Mokuno et al, 1992]. According to Kirino et al, NSE seems to be a marker of neuronal regeneration [Kirino et al, 1983].

NSE is also released from the brain to cerebrospinal fluid (CSF), as a result of an acute brain injury. Brain damage in patients undergoing open-heart surgery can result from brain ischaemia due to emboli (either air or thrombus) sent to the brain circulation through the ECC pump [McKibbin et al, 1976]. Embolic events caused by little pieces of plastic have been frequently reported in the past but currently, air, platelets thrombus and ECC agents are now considered as the commonest embolisation factors [Pearson et al, 1984]. It seems that the reduction of body temperature during ECC and the administration of barbiturates minimises the brain requirements for oxygen and protects it from the ischaemia that the emboli may cause [Steen et al, 1983]. Other drugs such as $\mathrm{Ca}^{++}$ blockers, 2-chloroadenosine [Evans et al, 1987] and phenytoin [Artru and Michenfelder, 1980] have also been used to reduce the incidence of postoperative brain damage following open-heart surgery. Among this neuroprotection process, it is very important to be able to identify in advance the patients in high-risk to develop brain dysfunction postoperatively. A large number of different agents and biomarkers have been occasionally used for the prognosis of postoperative brain damage during cardiac surgery. The S100 protein [Sellman et al, 1992], AK [Aberg et al, 1984], CK-BB [Taylor et al, 1980] are some of them used with moderate results.

\section{METHODS}

Our double blind study was based on a database of 100 patients admitted in two different departments; 50 of them in a Cardiac Surgery Department scheduled for open-heart surgery and 50 in a General Surgery Department for general surgery procedures respectively. The study protocol has been approved by the University's Scientific Ethics Committee. All patients were able to communicate with the investigators before the operation and an informed consent has been obtained in all cases. All of them had a previously unremarkable neurological and medical history upon their admission. The study was designed to measure and compare the level of NSE variance in the body serum of the patients before and after their scheduled operation. Thus, this patient population has been divided in two groups: The first group (group A), composed of 50 patients undergoing cardiac surgery because of coronary heart disease, and the second group (group B), composed of 50 patients also undergoing general surgery procedures. Among these two groups, we studied the correlation between the variance of the levels of NSE and the incidence of postoperative brain damage measured by the Hasegawa scale.

In order to measure NSE, we collected four blood samples $(3 \mathrm{~mL})$ at the following periods:

Group A:

- Sample 1: $24 \mathrm{~h}$ before the induction to anaesthesia

- Sample 2: before the entrance in the ECC

- Sample 3: immediately after the ECC

- Sample 4: $24 \mathrm{~h}$ after the operation.

Group B:

- Sample 1: $24 \mathrm{~h}$ before the induction to anaesthesia

- Sample 2: in the middle of the operation

- Sample 3: at the end of the operation

- Sample 4: $24 \mathrm{~h}$ after the operation.

The above blood samples were immediately centrifuged and the serum was frozen at $-70^{\circ} \mathrm{C}$ and stored for later NSE analysis, using a commercially available immunoradiometric assay kit. The anaesthetic drug protocol in both groups included the following agents: midazolam, vekouronioum, fentanyl, propofol and isoforenium, in doses depended on the body weight of the patients.

\section{RESULTS}

The final study included 92 patients (33 men and 59 women). 42 of them had undergone general surgery and 50 cardiac surgery. Eight patients were excluded from the study either because one of the blood samples was inappropriate or because the patient was unable or unwilling to be evaluated with the Hasegawa score postoperatively. The mean age was $61.7 \pm 11.6$ years (mean $\pm \mathrm{sd}$ ) and the time of stress period was $67 \pm 53.7$ min. The large standard deviation was due to the large sample range. The smallest recorded value was $2 \mathrm{~min}$ and the largest $314 \mathrm{~min}$. For each case, the level of NSE has been recorded four times. The first NSE level recorded $24 \mathrm{~h}$ before surgery, the second level just 
before surgery, the third immediately after surgery and the fourth $24 \mathrm{~h}$ following surgery. The mental ability of each patient, as we have already mentioned, was evaluated using the Hasegawa score, having the lowest and highest score recorded as 0 and 38 respectively and a mean value of $27.95 \pm 11$. 38. According to the results of our study, we have created the following algorithm:

$$
3.738 \times \text { sample } 3-4.221 \times \text { sample } 2>6.767
$$

If the above equation is fulfilled there is a very high possibility for postoperative brain dysfunction.

\section{Statistical analysis}

The normality assumption of the Hasegawa score distribution was tested with the use of the KolmogorofSmirnof test. Various nonparametric tests such as MannWhitney U-test, and Friedman's test were used, depending on the number of distributions tested. All the tests were at the level of significance $\mathrm{a}=0.05$. The $\mathrm{p}$-value of the Kolmogorof-Smirnof test is $\mathrm{p}=0.000$ which rejects the null hypothesis of Normality of the Hasegawa score. Using the above Hasegawa dementia scale, patients' population has been classified into four categories according to their mental stage (Table 1).

Friedman's test has been used for the testing of the equality of the distributions of the four NSE measurements. The power of the test is $p=0.000$, which means that the four distributions are not the same. The power of the Friedman test for the four NSE measurements within each of the four categories A, B, C, D, are 0.00 , $0.00,0.034,0.37$ respectively (Tables 2 and 3 ). It becomes obvious that the more severe the brain damage the stronger we reject the equality of the distributions. For the category D, where no brain dysfunction noted, the equality of the four distributions can not be rejected. Mean Hasegawa score for men and women is $25.61 \pm 7.61$ and $32.12 \pm 6.8$ respectively. The $\mathrm{p}$-value for the U-test is $\mathrm{p}=0.012$. The latter means that female patients take systematically higher score compared to the male ones. Spearman correlation coefficients between the age and the four NSE measurements were low, between 0.26 and 0.29 . In terms of the type of surgery, it was mentioned that the patients under general surgery developed almost no brain dysfunction. It has been observed that $75 \%$ of the patients under general surgery did not develop any brain damage. A percentage of $25 \%$ of them are at the limited stage $(\mathrm{C})$.

Then, we have grouped the four categories into two groups. The first group (group I) includes the categories A, B, C, in which the patients have developed brain dysfunction, while the second group (group II) includes the category $\mathrm{D}$, i.e. the patients who did not develop any type of brain dysfunction. The statistical features of the two groups are summarised in Table 4. It is obvious at the above table that the mean NSE level is the same in all four samples. The summary of the logistic regression is also presented in Table 5. The p-value of the Friedman's test is $p=0.31$, which does not reject the equality of the distributions. The same does not hold for Group B of the sick patients. The same test rejects the equality of the distributions of the four NSE measurements $(\mathrm{p}=0.000)$ (Fig. 1).

Table 1. Categories of patients according to Hasegawa score

\begin{tabular}{llllrrr}
\hline Category & Hasegawa score & Mental stage & Sample 1 & Sample 2 & \multicolumn{1}{c}{ Sample 3 } & Sample 4 \\
\hline A & $0-10$ & Dementia & $9.06 \pm 2.87$ & $10.25 \pm 3.13$ & $17.94 \pm 5.37$ & $17.15 \pm 7.15$ \\
B & $10.5-21.5$ & Partial dementia & $8.14 \pm 2.7$ & $9.38 \pm 2.62$ & $15.7 \pm 4.38$ & $16.36 \pm 6.17$ \\
C & $22-30.5$ & Limited & $5.62 \pm 1.64$ & $7.16 \pm 3.87$ & $9.36 \pm 5.06$ & $8.26 \pm 5.11$ \\
D & N1 & Normal & $9.16 \pm 4.74$ & $9.54 \pm 4.72$ & $9.19 \pm 4.26$ & $9.20 \pm 4.02$ \\
Mean & $27.9 \pm 11.8$ & & $8.7 \pm 4.2$ & $9.4 \pm 4.3$ & $11.05 \pm 5.5$ & $10.9 \pm 5.8$ \\
\hline
\end{tabular}

Table 2. Distribution of the patient's four categories in terms of the two types of surgery

\begin{tabular}{llll}
\hline Category & General surgery & Cardiac surgery & Total \\
\hline A & $0(25 \%)$ & $12(24 \%)$ & $12(13.1 \%)$ \\
B & $0(25 \%)$ & $10(20 \%)$ & $10(10.8 \%)$ \\
C & $21(25 \%)$ & $3(6 \%)$ & $24(26.1 \%)$ \\
D & $21(25 \%)$ & $25(50 \%)$ & $46(50 \%)$ \\
Total & $42(45.6 \%)$ & $50(54.4 \%)$ & $92(100 \%)$ \\
\hline
\end{tabular}

Table 3. Statistical features of the four NSE samples in terms of the kind of surgery

\begin{tabular}{llrr}
\hline & General surgery & Cardiac surgery & \multicolumn{1}{c}{ Total } \\
\hline Sample 1 & $8.66 \pm 5.44$ & $8.83 \pm 2.09$ & $8.7 \pm 4.2$ \\
Sample 2 & $8.77 \pm 5.50$ & $9.95 \pm 2.90$ & $9.4 \pm 4.3$ \\
Sample 3 & $7.77 \pm 4.46$ & $13.81 \pm 4.85$ & $11.05 \pm 5.5$ \\
Sample 4 & $8.09 \pm 4.56$ & $13.33 \pm 5.71$ & $10.9 \pm 5.8$ \\
\hline
\end{tabular}


Table 4. Statistical features of the group of patients who have (group I) and have not (group II) developed postoperative brain dysfunction

\begin{tabular}{llllrr}
\hline & Hasegawa score & Sample 1 & Sample 2 & Sample 3 & \multicolumn{1}{c}{ Sample 4 } \\
\hline Group I & $14.6 \pm 11.3$ & $7.2 \pm 2.8$ & $9.1 \pm 3.3$ & $14.9 \pm 5.9$ & $14.5 \pm 7.2$ \\
Group II & $34.3 \pm 1.6$ & $9.1 \pm 4.7$ & $9.5 \pm 4.7$ & $9.1 \pm 4.2$ & $9.2 \pm 4.0$ \\
\hline
\end{tabular}

Table 5. Summary of the logistic regression

\begin{tabular}{llll}
\hline & \multicolumn{2}{l}{ Predicted values } \\
\cline { 2 - 4 } & $\begin{array}{l}\text { Group A } \\
\text { (dementia) }\end{array}$ & $\begin{array}{l}\text { Group B } \\
\text { (healthy) }\end{array}$ & $\begin{array}{l}\text { Percentage of } \\
\text { correct prediction }\end{array}$ \\
\hline Recorded values & & & \\
Group A (dementia) & 24 & 1 & $96 \%$ \\
Group B (healthy) & 1 & 24 & $96 \%$ \\
Percentage of correct prediction & $96 \%$ & $96 \%$ & $96 \%$ \\
\hline
\end{tabular}

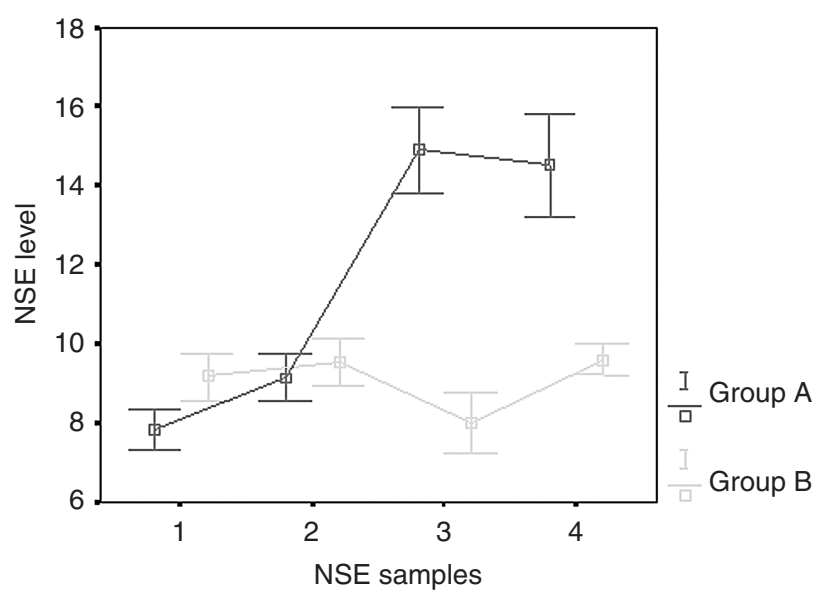

Figure 1. Mean values \pm sd for the four levels of NSE in the two group of patients.

In a graphic representation of this prognostic model (Fig. 2), patients at risk to develop brain ischaemia during cardiac surgery are distributed in the area above the line, while patients distributed in the area below the line are in significantly less risk to develop postoperative brain dysfunction. An attempt has been also made to construct a more convenient model by replacing the NSE levels by the time of stress period; however the result of this attempt was rather poor as the percentage of correct prediction was only $50 \%$ and consequently, it was abandoned.

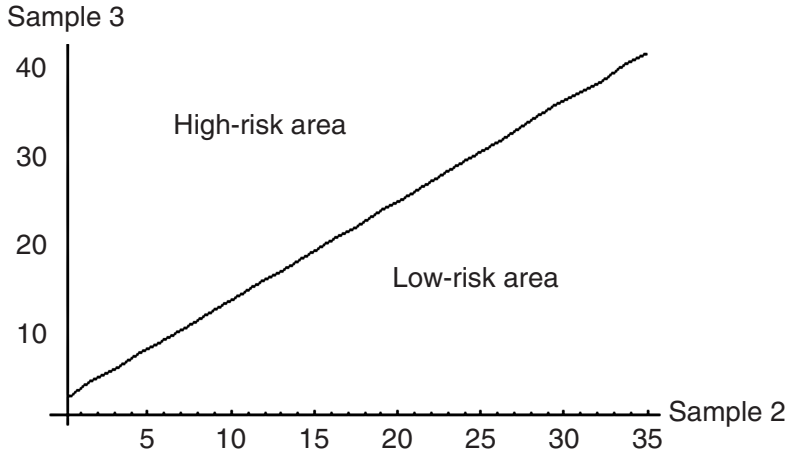

Figure 2. Geometric interpretation of the logistic model.

\section{DISCUSSION}

The aim of our study was to correlate the NSE blood serum levels during and immediately after surgery with the incidence of the postoperative brain damage, in patients undergoing general and open-heart surgery. Our hypothesis was that NSE is released as a result of brain tissue destruction.

There are previously published studies proposing the detection of the NSE isoenzyme in CSF, as the preferable method in terms of diagnostic accuracy. However, there are several limitations regarding the use of this method. A number of technical problems, such as the patient position or the multiple punctures of the dura mater necessary to obtain a series of samples in different times, make an attempt to take CSF samples during the operation difficult or even impossible. Thus, all the available 
published series using the above method, detect the NSE levels in CSF after the end of the operation; however, in this situation, the most crucial samples during the operation are lost. Alternatively, some authors recommend the detection of the NSE levels in blood serum as an accurate and effective method for the prognosis of the postoperative brain dysfunction [Roine et al, 1989].

A review of the published literature reveals a significant variance in the conclusions concerning the relationship between the NSE levels and the postoperative brain dysfunction.

Johnsson et al [1995, 1996] found high NSE levels in cardiosurgical patients but not critical neurological outcome in the postoperative period. Sellman et al [1992] found no relationship between high NSE levels and postoperative brain damage. Isgro et al [1997], suggested that high NSE levels after open-heart surgery are followed by brain damage installation and Hardemark et al in 1988 and 1989, found a quantitative relationship between the NSE levels and the severity of the postoperative brain damage [Hardemark et al, 1988, 1989].

According to older studies [Frank et al, 1972] the incidence of the postoperative neurological complications following cardiac surgery can be described by a $0-100 \%$ scale. In most recent publications, Adrian et al [1995], found neurological complications ranging from 1 to $57 \%$. In our study, the incidence of neurological complications was $44 \%$ for the open-heart surgery group and not significant for the general surgery group. The serum NSE levels among the four different blood samples were found the same in the general surgery group, but this was not the case in the open-heart surgery group (p-values: 0.612 and 0.000 , median Hasegawa score: $33.6 \pm 2.72$ and $23.14 \pm 13.51$ respectively).

The current trend regarding open-heart procedures, is towards early mobilisation and shorter hospitalisation, especially concerning the postoperative period patients spend in the ICU. In the presented study, we have collected three different blood samples for each individual patient during the operation, as we have assessed this period as the most crucial in the prognosis of postoperative brain dysfunction. To further support this assessment, the highest NSE serum levels have been found during the ECC. Thus, by evaluating the NSE levels at the time 2 and 3 of the operation, it may be possible to identify the patients in high-risk for postoperative brain damage.

Savageau et al [1982], identified a positive relation between patient's age and postoperative brain damage. We have also found a similar correlation between these two parameters. Furthermore, sex was found another factor influencing brain dysfunction following cardiac surgery. Men in our study had a median Hasegawa score of $25.61 \pm 7.61$, while the mean Hasegawa score for women was $32.12 \pm 6$. NSE serum levels at the same time were lower for female than for male patients. These results indicate that women are expected to have better neurological outcome in the postoperative period, but this cannot be an absolute conclusion since the number of female patients in the open-heart surgery group was small.

Another relationship was also found between the duration of the ECC and the installation of postoperative brain dysfunction in our series. It is obvious that when the time of the ECC is prolonged, the possibility for the occurrence of events that may influence brain function becomes higher and this must be the most probable explanation for this correlation. Similar results and conclusions have been also presented in other published studies [Isgro et al, 1997; Sellman et al, 1992].

A number of previously reported series [Aberg et al, 1984; Sellman et al, 1992; Zelter et al, 1986], have examined the role of various other enzymes as indicators of the postoperative brain dysfunction is an intracellular enzyme that can be detected in the red blood cells as well as in CSF after cerebral ischaemia. The detection of high levels of AK has a positive correlation to brain dysfunction after ECC but the presence of AK in serum may be also the result of haemolysis and thus, a cause of false positive results. The detection of protein $\mathrm{S} 100^{2}$ [Sellman, 1992] may also be used as an indicator of the postoperative brain damage, but it is a more expensive and a less sensitive method. CK-BB [Taylor et al, 1980] is a predictive biochemical marker used by some investigators, but has not proved as reliable as NSE as it creates frequent cross-reactions to other isoenzymes. Eventually, it seems that the detection of blood serum NSE is the most accurate and reliable factor available for early prognosis of brain dysfunction following open-heart surgery [Johnsson et al, 1996].

\section{CONCLUSION}

The results of our double blind study shows that blood serum NSE levels, particularly immediately before and after the ECC, is a sensitive, inexpensive and safe method that may contribute significantly in the early prognosis of postoperative brain dysfunction. Further studies focusing on the predictive role of the NSE levels in different types of operations with a high incidence of perioperative brain ischaemia, such as thoracic aorta or brain aneurysms, will be necessary for evaluation of its efficacy as a prognostic factor in a wider range of performances. 


\section{REFERENCES}

Aberg T, Ronguist G, Tyden H, et al. Adverse effects on the brain in cardiac operations as assessed by biochemical, psychometric, and radiologic methods. The Journal of Thoracic Cardiovascular Surgery 1984; 87: 99-105.

Adrian L, Radinowicz N, Jorge D, et al. Neuron-Specific Enolase is increased after non-convulsive status epilepticus. Epilepsia 1995; 136: 302-307.

Artru A, Michenfelder J. Cerebral protective, metabolic and vascular effects of phenytoin. Stroke 1980; 11: 377-382.

Chang SF, Suh JW, Choi-JW, et al. Diagnostic pitfalls of Merkel cell carcinoma and dramatic response to chemotherapy. Journal of Dermatology 1998; 25: 322-331.

Evans MC, Swan JH, Meldrum BS. An adenosine analogue, 2 chloroadenosine, protects against long term development of ischemic cell loss in the rat hippocampus. Neuroscience Letters 1987; 83: 287-292.

Fossa SD, Klepp O, Paus E. Neuron specific enolase : a serum marker in seminoma? British Journal of Cancer 1992; 65: 297-299.

Frank KA, Heller SS, Kornfeld DS, et al. Long term effects of open heart surgery on intellectual functioning. The Journal of Thoracic Cardiovascular Surgery 1972; 64: 811-815.

Hardemark HG, Ericsson N, Kotwica Z, et al. S-100 Protein and NSE in CSF after experimental traumatic or focal ischemic brain damage. Journal of Neurosurgery 1989; 71: 727-731.

Hardemark HG, Persson L, Bolander $\mathrm{HG}$, et al. NSE is a marker of cerebral ischemia and infarct size in rat cerebrospinal fluid. Stroke 1988; 19: 1140-1144.

Isgro F, Schmidt C, Pohl P, Saggau W. A predictive parameter in patients with brain related complications after cardiac surgery? European Journal of Cardiothoracic Surgery 1997; 11 : 640-644.

Ishiguro Y, Kato K, Ito T, et al. Nervous system-specific enolase in serum as a marker for neuroblastoma. Pediatrics 1983; 72: 696-700.

Johnsson P. Markers of cerebral ischemia after cardiac surgery. Journal of Cardiothoracic and Vascular Anesthesia 1996; 10: 120-126.

Johnsson P, Lundqvist C, Lindgren A, et al. Cerebral complications after cardiac surgery assessed by S-100 and NSE levels in blood. Journal of Cardiothoracic and Vascular Anesthesia 1995; 9: 1-7.

Johnsson P, Lundqvist C, Lindgren A, et al. Cerebral complications after cardiac surgery assessed by S-100 and NSE levels in blood. Journal of Cardiothoracic and Vascular Anesthesia 1996; 9: 694-699.

Ke Ping X, Shang Lian L, Chuo N. Immunohistochemical evidence of neuronal and glial differentiation in retinoblastoma. British Journal of Ophthalmology 1995; 79: 771-776.

Kirino T, Brightman MW, Oertel WH, et al. Neuron-specific enolase as an index of neuronal regeneration and reinnervation. Neuroscience 1983; 3 : 915-923.

Lorenz J, Dippold W. Neuron-specific enolase: a marker for malignant melanoma. Journal of National Cancer Institute 1989; 81: 1754-1755.

Massaron S, Seregni E, Luksch R, et al. Neuron-specific enolase evaluation in patients with neuroblastoma. Tumour Biology 1998; 19: 261-268.

McKibbin DW, Gott VL, Hutckin S, et al. Fatal cerebral atheromatous embolization after cardiopulmonary bypass. Journal of Thoracic and Cardiovascular Surgery 1976; 71: 741-745.
Mokuno K, Yasuda T, Sugimura K, et al. Cerebrospinal fluid neuronspecific enolase (NSE) and S-100b protein in Guillain Barre syndrome their relations to prognosis. Rin Shin 1992; 32: 535-537.

Moore BW, McGregor T. Chromatographic and electrophoretic fractionation of soluble proteins of brain and liver. Journal of Biological Chemistry 1965; 240: 1647-1653.

Pearson DT, Holden MP, Poslad SS, et al. A clinical evaluation of the gas transfer characteristics and gaseous microemboli production of two bubble oxygenators. Life Support Systems 1984; 2: 252-256.

Roine RR, Somer H, Kaste M, et al. Neurological outcome after out-ofhospital cardiac arrest. Prediction by cerebrospinal fluid enzyme analysis. Archives of Neurology 1989; 46: 753-756.

Savageau JA, Stanton BA, Jenkins CD, Frater RW. Neuro-psychological dysfunction following elective cardiac operation: Early assessment. Journal of Thoracic and Cardiovascular Surgery 1982; 84: 595-600.

Sellman M, Ivert T, Ronquist G, Caesarini $\mathrm{K}$, et al. Central nervous system damage during cardiac surgery assessed by 3 different biochemical markers in cerebrospinal fluid. Scandinavian Journal of Thoracic and Cardiovascular Surgery 1992; 26: 39-45.

Steen PA, Newberg LA, Milde JH, Michenfelder JD. Hypothermia and barbiturates: individual and combined effects on canine cerebral oxygen consumption. Anesthesiology 1983; 58: 527-532.

Taylor KM, Devlin BJ, Mittra SM, et al. Assessment of cerebral damage during open heart surgery. A new experimental model. Scandinavian Journal of Thoracic and Cardiovascular Surgery 1980; 14: 197-203. 
Zeltzer PM, Parma AM, Dalton A, et al. Raised neuron-specific enolase in serum of children with metastatic neuroblastoma. Lancet 1983; 2: 361-363.

Zeltzer PM, Schneider SL, Marangos PJ, Zweig MH. Differential expression of neural isozymes by human medulloblastomas and gliomas and neuroectodermal cell lines. Journal of National Cancer Institute 1986; 77 : 625-631.

Zerri P, Bodemer M, Racker S, et al. Cerebrospinal fluid concentration of neuron-specific enolase in diagnosis of Creutzfeldt Jacob disease. Lancet 1995; 24: 1609-1610.
Zomzely-Neurath CE. Enolase. In: Lajtha A (ed.). Handbook of Neurochemistry (2nd edn). New York: Plenum, 1983, 403-433. 